\title{
Compensation of Intra-Frame Head Motion in PET Data with Motion Corrected Independent Component Analysis (MCICA)
}

\author{
Martin J. McKeown ${ }^{* 1,3,4}$, Marwa Gadala ${ }^{2}$, and Rafeef Abu-Gharbieh ${ }^{2,4}$ \\ Departments of Medicine ${ }^{1}$ and Electrical and Computer Engineering ${ }^{2}$, Pacific Parkinson's Research \\ Centre $^{3}$, Brain Research Centre ${ }^{4}$, University of British Columbia \\ mmckeown@interchange.ubc.ca ,\{mgadala, rafeef $\} @$ ece.ubc.ca
}

Keywords: Positron Emission Tomography, Motion Corrected Independent Component Analysis.

\section{ABSTRACT}

Independent Component Analysis (ICA) has proved a powerful exploratory analysis method for fMRI. In the ICA model, the fMRI data at a given time point are modeled as the linear superposition of spatially independent (and spatially stationary) component maps. The ICA model has been recently applied to positron emission tomography (PET) data with some success (Human Brain Mapping 18:284-295(2003), IEEE Trans. BME, Naganawa et al, in press). However, in PET imaging each frame is, in fact, activity integrated over a relatively long period of time, making the assumption that the underlying component maps are spatially stationary (and hence no head movement has taken place during the frame collection) very tenuous. Here we extend the application of the ICA model to ${ }^{11} \mathrm{C}$-methylphenidate PET data by assuming that each frame is actually composed of the superposition of rigidly transformed underlying spatial components. We first determine the "noisy" initial spatially independent components of a data set under the erroneous assumption of no intra or inter-frame motion. Aspects of the initial components that reliably track spatial perturbations of the data are then determined to produce the motion-compensated components. Initial components included ring-like spatial distributions, indicating that movement corrupts the statistical properties of the data. The final intra-frame motion-compensated components included more plausible symmetric and robust activity in the striatum as would be expected compared to the raw data and the initial components. We conclude that 1) intra-frame motion is a serious confound in PET imaging which affects the statistical properties of the data and 2) our proposed procedure ameliorates such motion effects.

\section{INTRODUCTION}

Head motion remains a serious confound for functional brain imaging [6]. Often similar approaches are used to correct for motion in fMRI and Positron Emission Tomography (PET). In these cases, motion correction is typically done by optimizing some cost function quantifying the match between each frame in the series and a "reference image" that is often the first volume in the series, or perhaps the mean image. In case of assuming that a motion-corrected volume is identical to the "reference volume" plus white Gaussian noise, the maximum likelihood estimates of the motion parameters are obtained by minimizing the sum of squared differences in the image intensities. Here we argue that the key assumption of square of difference-based measures, namely that all signal intensity differences between the reference volume and a motion-corrupted volume are wholly due to motion plus white Gaussian noise is inappropriate since brain imaging data include dynamically changing brain signals as well as other sources of variability with nonrandom spatial and temporal structure. Thus simulations demonstrate that sum of squared difference methods can be "fooled" into inferring motion when dynamically varying activations are incorporated into an absolutely motionless time-series [4].

\footnotetext{
${ }^{*}$ M31, Purdy Pavilion, University Hospital, UBC Site, 2221 Wesbrook Mall, Vancouver, British Columbia V6T 2B5 Canada. Tel. (604) 822-7516, Fax. (604) 822-7866, mmckeown@interchange.ubc.ca
} 


\subsection{Motion-Corrected Independent Component Analysis (MCICA)}

We have recently proposed the Motion-Corrected Independent Component Analysis (MCICA) method as a means to mitigate the effects of motion on fMRI $[8,9]$. In the ICA model, the fMRI data at a given time point are modeled as the linear superposition of spatially independent (and spatially stationary) component maps. The ICA model has been recently applied to PET data with some success $[14,15]$.

When ICA is applied to fMRI or PET data, the data are usually expressed as a time-by-voxel matrix $\boldsymbol{X}$ (i.e. the measurements of all voxels within a volume at a single time point are concatenated as a row vector), and are modeled as the linear sum of the contributions from several spatially independent processes $[10,11,12,13]$ :

$X=A S$

where $\boldsymbol{X}=\left[\mathbf{x}_{1}, \ldots, \mathbf{x}_{\mathrm{n}}\right]^{T}, \boldsymbol{A}=\left[\mathbf{a}_{1}, \ldots, \mathbf{a}_{\mathrm{n}}\right]$, and $\boldsymbol{S}=\left[\mathbf{s}_{1}, \ldots, \mathbf{s}_{\mathrm{n}}\right]^{T}$

Here $\mathbf{x}_{\mathrm{i}}(i=1, \ldots, n)$ is the transpose of the row vector collecting the fMRI/PET observations for the $\mathrm{i}^{\text {th }}$ frame, $\mathbf{a}_{\mathrm{k}}(k=1, \ldots$, $n)$ is a column vector representing the activation waveform for the $\mathrm{k}^{\text {th }}$ process and $\mathbf{s}_{\mathrm{k}}(k=1, \ldots, n)$ is a column vector representing the activation map for the $\mathrm{k}^{\text {th }}$ process. Each column of $\mathbf{X}$ and $\mathbf{S}$ can be viewed as independent realizations of the random vectors $\mathbf{x}=\left[x_{l}, \ldots, x_{n}\right]^{T}$ and $\mathbf{x}=\left[s_{l}, \ldots, s_{n}\right]^{T}$ respectively. The objective of ICA is to find the unmixing matrix $\mathbf{W}$ (the inverse of $\mathbf{A}$, with possibly scaled and permuted rows) to achieve the independent component map estimation $\widetilde{\mathbf{s}}=\mathbf{W} \mathbf{x}$. . With the Infomax algorithm [1], W is estimated by maximizing H[y] where H[.] denotes the Shannon (joint) entropy of a random vector, and

$\mathbf{y}=\mathrm{g}(\widetilde{\mathbf{s}})=\mathrm{g}(\mathbf{W} \mathbf{x})$

Here $g(\widetilde{\mathbf{s}})=\left[g_{l}\left(\widetilde{\mathbf{s}}_{1}\right), \ldots, g_{n}\left(\widetilde{\mathbf{s}}_{\mathrm{n}}\right)\right]^{T}$ and the nonlinear function $g_{k}().(k=1, \ldots, n)$ is typically the logistic function, $y_{k}=g_{k}\left(\tilde{s}_{k}\right)=\frac{1}{1+e^{-\tilde{s}_{k}}}$,

for fMRI \& PET signals with super-Gaussian distributions. It can be shown that [1],

$$
H[\mathbf{y}]=H[\mathbf{x}]+E\{\log |J|\}
$$

where $E\{$.$\} denotes statistical expectation and |\mathbf{J}|$ is the absolute value of the determinant of the Jacobian matrix of transformation from $\mathbf{x}$ to $\mathbf{y}$.

The core idea of the MCICA algorithm is based on the observation that motion effects within both PET and fMRI data will result in an increase in $H[\mathbf{x}]$ and a simultaneous decrease in $H[\mathbf{y}]$ in Eq. (4). Specifically, misalignment of some time point with respect to other time points in the fMRI data matrix $\boldsymbol{X}$ tends to decrease the higher-order mutual information $M[\mathbf{x}]$ among the elements of the nominal random vector $\boldsymbol{x}$ (i.e. among the rows of $\boldsymbol{X}$ ) and hence increases the joint entropy of the data, $H[\mathbf{x}]$. Note that when considering only two time points in the fMRI time-series, the 2variate joint entropy $H[\mathbf{x}]$ defaults to the equivalent of 2-variate mutual information $M[\mathbf{x}]$, a well-validated objective function to be (mini)maximized for image registration [Boes, 999 \#18;Collignon, 1995 \#16;Studholme, 1995 \#17]. In addition to the increase in $H[\mathbf{x}], H[\mathbf{y}]$ tends to simultaneously decrease for motion-corrupted data for two reasons (refer to [9] for a detailed explanation): 1) motion-related ICA component(s) tend to be generated, and these have an accumulative distribution function that cannot be well approximated by the logistic function, decreasing the marginal entropy $H\left[y_{k}\right]$ of the output $\boldsymbol{y}$; and 2) the extracted ICA components tend to become more dependent for motioncorrupted data, which potentially increases the mutual information $M[\mathbf{y}]$ among the elements of $H[\mathbf{y}]$. The joint entropy, $H[\mathbf{y}]$, which is related to the sum of the marginal entropy,

$\sum_{k=1}^{n} H\left[y_{k}\right]$ and the mutual information $M[\mathbf{y}]$ with opposite sign, therefore tends to decrease for the data corrupted with motion.

Since $H[\mathbf{x}]$ increases and $H[\mathbf{y}]$ decreases with motion, the entropy difference, $E\{\log |\mathbf{y}|\}$, is strongly affected by motion and can therefore be used as an objective function to be maximized for movement correction within the framework of ICA. The proposed objective function can be calculated as: 
$E\{\log |J|\}=\log |\operatorname{det} \mathbf{W}|+E\left\{\sum_{k=1}^{n} \log y_{k}^{\prime}\right\}$

where

$y_{k}^{\prime}=\frac{\partial y_{k}}{\partial \tilde{s}_{k}}=y_{k}\left(1-y_{k}\right)$

Critically, the optimization criterion, $E\{\log |\mathbf{y}|\}$, is assessed on an ensemble of volumes. Intuitively, since using two reference images improves registration performance [2], extending this to an arbitrary number of reference images in a computationally tractable manner has the potential to significantly enhance registration performance. If the ensemble of (assumed motionless) volumes chosen to compute $E\{\log |\mathbf{y}|\}$ capture the major sources of variability in the data, then signal changes due to brain activations will be implicitly modeled and will not interfere with motion estimation. Specifically, if $E\{\log |\mathbf{y}|\}$ is maximized with respect to the spatial transformation of different volumes, the variability of voxels' time courses will in effect be partitioned into "motion", as captured by the spatial transformations, and "nonmotion" variability, captured in $E\{\log |\mathbf{y}|\}$.

This implicit yet flexible modeling of non-motion related signal changes in the proposed objective function is the key feature that helps MCICA achieve robust performance in motion correction of fMRI data that contain dynamically changing brain signals. Another significant advantage of using $E\{\log |\mathbf{y}|\}$ as the objective function for motion mitigation is that its gradient can be assessed analytically, which allows for incorporation of gradient information for maximization process [9].

The MCICA algorithm requires that a given motion-corrupted volume is first rotated and translated to create a set of $m_{r}$ basis images, each image denoted by a column vector $\mathbf{r}_{i}\left(i=1, \ldots, m_{r}\right)$ and collected into a matrix $\boldsymbol{R}=\left[\boldsymbol{r}_{l}, \ldots, \boldsymbol{r}_{\mathrm{mr}}\right]^{T}$.. The final registered image is then created by taking an appropriate linear combination of these basis images. The MCICA algorithm thus determines the unit-length $m_{r} \mathrm{x} 1$ motion-compensation vector $\boldsymbol{p}$ containing weights for each of the $m_{r}$ basis images in $\boldsymbol{R}$, together with the unmixing matrix $\boldsymbol{W}$, so that the objective function, $E\{\log |\mathbf{y}|\}$, is maximized for the registered data matrix $\boldsymbol{X}^{\mathrm{r}}$,:

$$
\mathbf{X}^{r}=\left[\begin{array}{llll}
\mathbf{R}^{T} \mathbf{p} & \mathbf{x}_{2} & \cdots & \mathbf{x}_{q}
\end{array}\right]^{T}
$$

where $\boldsymbol{X}_{\mathrm{i}}(i=2, \ldots, q)$ represents the subset of volumes that act as the "reference volumes" for the fMRI time-series (explained later), and the first time point is now replaced by $\boldsymbol{R}^{T} \boldsymbol{p}$, a linear sum of the $m_{r}$ basis images in $\boldsymbol{R}$ weighted by the motion-compensation vector $\boldsymbol{p}$ to approximate the registered image for the first time point. The final registered image then can be obtained as the linear combination of the basis images weighted by the estimated motioncompensation weights, subject to rescaling and baseline offset to the same standard deviation and mean of the original image.

However, in the PET case, each frame is, in fact, activity integrated over a relatively long period of time, making the assumption that the underlying component maps are spatially stationary (and hence no head movement has taken place during the frame collection) very tenuous.

\subsection{Maximum Autocorrelation Factor (MAF) Analysis}

An alternate method to decompose multivariate data is to search for components which have a large amount of spatial autocorrelation $[3,5,7,16]$. This is based on the assumption that "interesting" components have a large spatial autocorrelation, while noise will tend to have a small autocorrelation. MAF can be considered as a variant of canonical correlate analysis (CCA) [5].

\subsubsection{Canonical Correlate Analysis (CCA)}

CCA can be summarized as follows: consider two multivariate random variables, $\boldsymbol{a}$ and $\boldsymbol{b}$. In CCA, we seek two vectors, $\boldsymbol{w}_{\boldsymbol{a}}$ and $\boldsymbol{w}_{\boldsymbol{b}}$, such that: 
$\rho=\operatorname{Corr}\left\{\boldsymbol{w}_{\boldsymbol{a}}(\boldsymbol{a}-\overline{\boldsymbol{a}}), \boldsymbol{w}_{\boldsymbol{b}}(\boldsymbol{b}-\bar{b})\right\}$

is maximized. The value $\rho$ is referred to as the largest canonical correlation. The $\mathrm{i}^{\text {th }}$ Canonical Correlate is the linear combination that exhibits the highest correlation subject to it being uncorrelated to the previous Canonical Correlates [3].

\subsubsection{MAF Applied to Images}

Maximum autocorrelation factor (MAF) analysis was originally proposed as an alternative transformation of multivariate spatial imagery to the widely used Principal Components Analysis (PCA) transform by Switzer [16]. In the MAF analysis one seeks a transformation that maximizes the autocorrelation between neighboring observations (i.e. pixels). By building the additional information of the structure of the observations into the model, i.e. that interesting signal exhibits high autocorrelation relative to noise, the signal decompositions will better separate the interesting signal from the noise especially in cases where the noise variance is larger than the signal variance.

In MAF analysis, we chose $\boldsymbol{a}$ and $\boldsymbol{b}$ from (7) such that $\boldsymbol{a}=\boldsymbol{X}(\mathrm{x}, \mathrm{y}, \mathrm{z})$; and $\boldsymbol{b}=\mathrm{T}_{\Delta}\left(\boldsymbol{X}(\mathrm{x}, \mathrm{y}, \mathrm{z})\right.$ ), where $\mathrm{T}_{\Delta}()$ is a typically "small" affine transformation. We note that MAF factors are invariant under an affine transformation [7].

He we suggest that spatially independent components computed from a set of PET images will be degraded by intraframe motion. Rather than using MAF to extract components from the PET images, which relies on pairwise correlation between images, we propose using a mutual information criterion. Also, since each component is derived from an ensemble of images, we suggest that it is more appropriate to maximize the mutual information between a linear combination of spatially transformed components, and the original data that has been shifted a small amount. Stated in this way, an effective MAF-type method for IC denoising can be implemented with the MCICA method.

\section{PROPOSED METHOD}

ICA is first applied to a PET data set using (1), giving us maximally spatially-independent components $s$ (which includes intraframe motion). Following the Maximum Autocorrelation Factor (MAF) approach we transform the data with a "small" transformation,

$\boldsymbol{X}_{\Delta}=\boldsymbol{T}_{\Delta \mathrm{o}}[X]$.

We assume that the 'true' underlying spatial distribution of the spatially independent components will be invariant under a coordinate change, $\boldsymbol{T}_{\Delta}[$.].

We then create the 'R' matrix for the first component by choosing multiple affine transformations of the IC:

$\mathrm{R}=\left[\boldsymbol{T}_{\Delta 1}\left[\boldsymbol{C}_{1}\right], \boldsymbol{T}_{\Delta 2}\left[\boldsymbol{C}_{1}\right], \boldsymbol{T}_{\Delta 3}\left[\boldsymbol{C}_{1}\right] \ldots, \boldsymbol{T}_{\Delta \mathrm{q}}\left[\boldsymbol{C}_{1}\right]\right]^{T}$

We ensure that the $\mathrm{T}_{\Delta}$ 's span the transformation applied to the original data, $\mathrm{T}_{\Delta \mathrm{o}}[]$.

Calculation of $\boldsymbol{p}$ with MCICA will then result in a linear combination of 'noisy' components that have maximal mutual information with $\mathrm{T}_{\Delta}[\mathrm{X}]$. Since the underlying 'true' components, $\mathrm{D}$ will be invariant under an affine transformation; $\boldsymbol{p R}$ will be an approximation for $\mathrm{T}_{\Delta}\left[\mathrm{D}_{l}\right]$. $\mathrm{D}_{1}$ can then be recovered by: $\mathrm{T}_{\Delta}^{-1}[\boldsymbol{p} \boldsymbol{R}]$. This process is repeated for $\boldsymbol{D}_{i}, i=(2, \ldots$, $n$ ). The time courses of the components $\mathrm{D}$, can then be estimated by:

$\boldsymbol{A}=\left(\boldsymbol{D} \boldsymbol{D}^{T}\right)^{-1} * \boldsymbol{X}$

We applied the above method to a single scan using ${ }^{11} \mathrm{C}$-methylphenidate tracer. Each slice obtained had a matrix size of $128 \times 128$, and a total of 31 slices were collected. A total of 16 volumes were collected. Voxels outside the brain (determined by mean activity) were excluded from further analysis. 
Infomax ICA was used to separate the data into spatially independent, "noisy" components. The original data were transformed by: $\mathrm{X}_{\Delta}=0.5, \mathrm{Y}_{\Delta}=-0.5, \mathrm{Z}_{\Delta}=0.5, \mathrm{X}_{\theta \Delta}=0.5, \mathrm{Y}_{\theta \Delta}=-0.5 \mathrm{Z}_{\theta \Delta}=0.5$, where the first 3 variables respond to spatial shifts (in voxels) and the latter represent rotations around the respective axes (in degrees).

Each ' $\mathrm{R}$ ' matrix (eqn (9)) was created by taking a component $\boldsymbol{s}_{i}$, and additionally rotating the component $[-5,-2,2,5]$ degrees around each axis and also shifting the data $[-2,2]$ voxels in each of the 3 axes, resulting in 73 rows. The vector ' $p$ ' was then calculated using the MCICA method previously described.

\section{RESULTS:}

The initial 'noisy' ICA components included ones with ring-like spatial distributions indicative of movement (Figure 1). In addition, the initial "noisy" ICA components "fragmented" activity within the striatum across at least 2 different components (Figure 2, top and middle panels). After the proposed method was applied, the striatal activity became more robust (Figure 2, bottom panel).
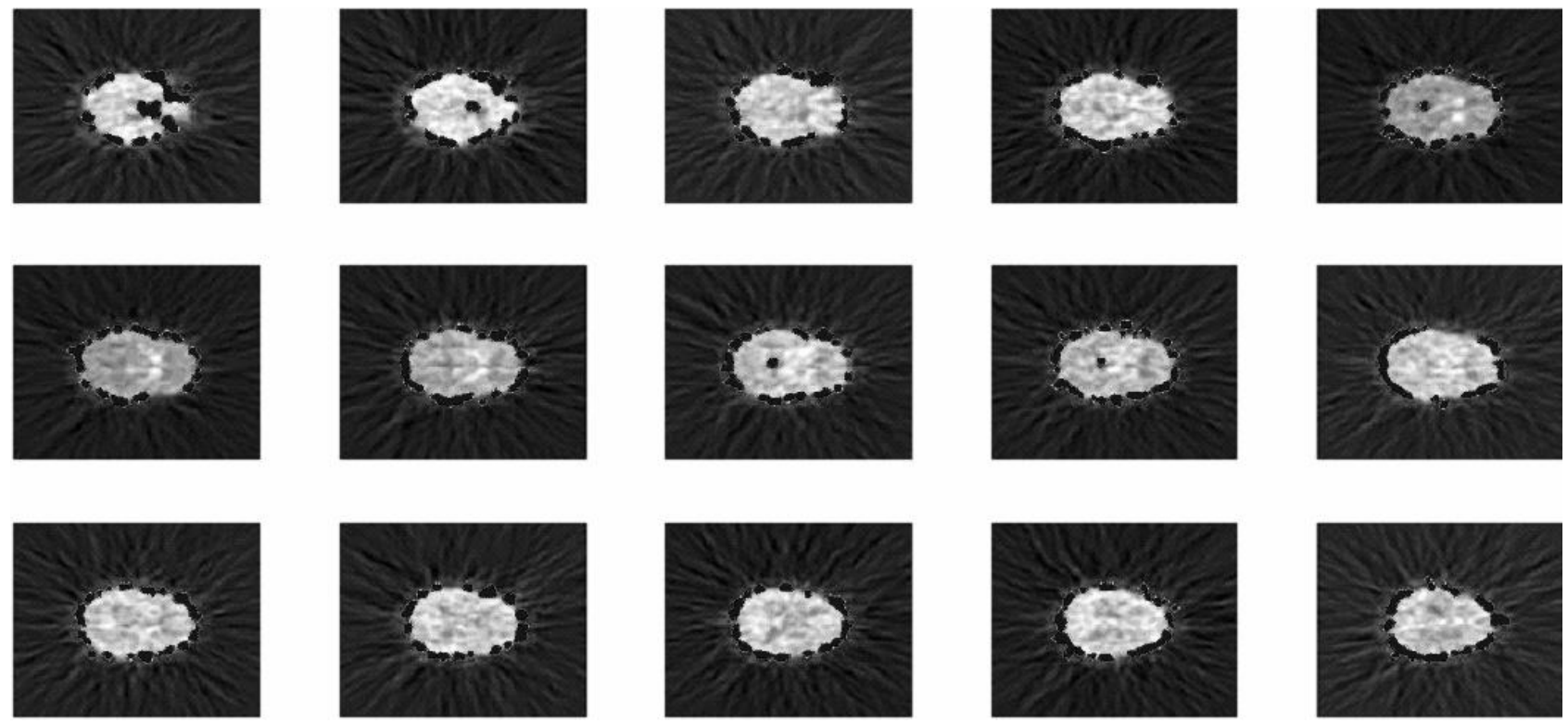

Figure 1. The initial 'noisy' ICA components (shown in dark black).

\section{CONCLUSION:}

Currently PET motion correction methods are unable to deal with intraframe motion. In this paper, we have presented a first step towards solving this problem by extending the ICA model recently proposed for the analysis of PET data to implicitly include motion compensation.

We proposed a decomposition technique that intially utilizes regular ICA to estimate motion-corrupted independent components (ICs ) of the image data. We then, inspired by the MAF analysis paradigm, further process the resulting motion-corrupted ICs by identifying components that have maximal mutual information with all subsequent images in the data set.

Our initial results illustrate how regular ICA components of PET data display ring-like spatial distributions typically indicative of movement. Our motion-compensated ICA components on the other hand seem to consist of more plausible spatially coherent components comprising contiguous activity maps.

Though initial indications show that this novel motion correction scheme for compensating for intra-frame motion in PET data is promising, further work is required to determine the range of data sets where this is is applicable. 


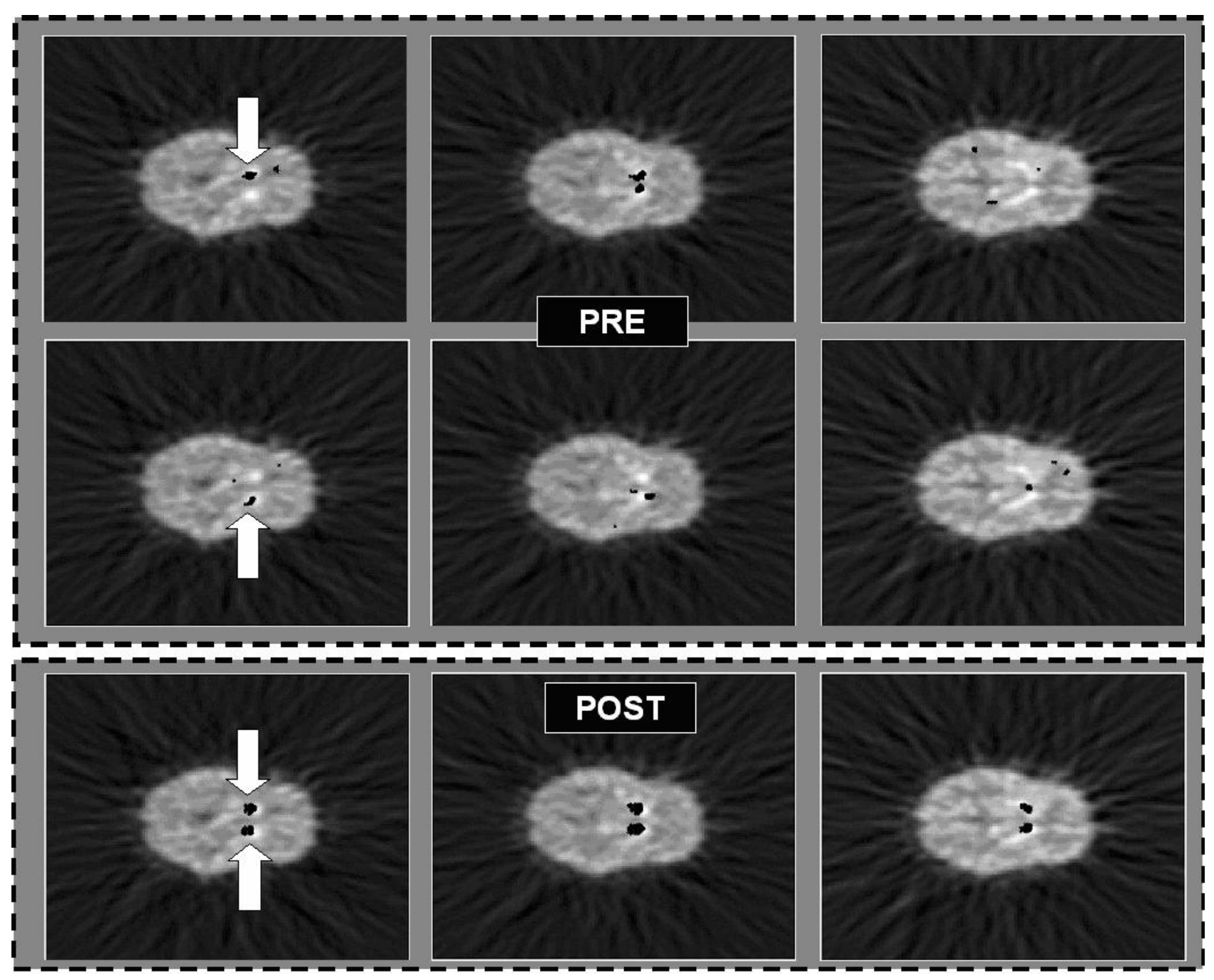

Figure 2.

(Top \& middle panel): Initial "noisy" ICA components include uptake in the striatum, as expected, but the spatial distribution was fragmented at least across 2 different components.

(Bottom panel): A motion-compensated ICA component now clearly coalesces the fragmented components into a spatially coherent activity map incorporating both the left and right striatum, as would be expected from prior studies incorporating $11 \mathrm{C}$ methylphenidate.

\section{ACKNOWLEDGEMENTS}

The authors are grateful to Vesna Sossi, PhD, for providing us with the PET data, and Ms. Siobhan McCormick for some assistance in pre-processing the data. 


\section{REFERENCES}

1. A.J. Bell and T.J. Sejnowski, "An information-maximization approach to blind separation and blind deconvolution," Neural Comput, vol. 7, no. 6, 1995, pp. 1129-59.

2. J.L. Boes and C.R. Meyer, "Multi-variate Mutual Information for Registration," Lecture Notes in Computer Science, vol. 1679, 1999, pp. 606-612.

3. M. Borga and H. Knutsson, A Canonical Correlation Approach to Blind Source Separation, Technical report, Linkoping University, 2001.

4. L. Freire, A. Roche, and J.F. Mangin, "What is the best similarity measure for motion correction in fMRI time series?," IEEE Transactions on Medical Imaging, vol. 21, no. 5, 2002, pp. 470-84.

5. A.A. Green, M. Berman, P. Switzer, and M.D. Craig, "A Transformation for Ordering Multispectral Data in Terms of Image Quality and Implications for Noise Removal," IEEE Transactions on Geoscience and Remote Sensing, vol. 26, 1998, pp. 65-74.

6. J.V. Hajnal, R. Myers, A. Oatridge, J.E. Schwieso, I.R. Young, and G.M. Bydder, "Artifacts due to stimulus correlated motion in functional imaging of the brain," Magnetic Resonance in Medicine, vol. 31, no. 3, 1994, pp. 283-91.

7. R. Larsen, "Decomposition using maximum autocorrelation factors," Journal of Chemometrics, vol. 16, 2002, pp. 427-435.

8. R. Liao, J. Krolik, and M.J. McKeown, "Isolation and Minimization of Effects of Motion on fMRI Using Multiple Reference Images," in Proceedings of 2004 IEEE International Symposium on Biomedical Imaging:From Nano to Macro, Arlington, VA, 2004.

9. R. Liao, J.L. Krolik, and M.J. McKeown, "An Information-Theoretic Criterion for Intrasubject Alignment of FMRI Time Series: Motion Corrected Independent Component Analysis," IEEE Transactions on Medical Imaging, vol. 24, 2005, pp. 29-44.

10. M.J. McKeown, L.K. Hansen, and T.J. Sejnowski, "Independent component analysis of functional MRI: What is signal and what is noise?," Current Opinions in Neurobiology, vol. 13, 2003, pp. 620-629.

11. M.J. McKeown, T.P. Jung, S. Makeig, G. Brown, S.S. Kindermann, T.W. Lee, and T.J. Sejnowski, "Spatially independent activity patterns in functional MRI data during the stroop color-naming task," Proceedings of the National Academy of Sciences of the United States of America, vol. 95, no. 3, 1998, pp. 803-10.

12. M.J. McKeown, S. Makeig, G.G. Brown, T.P. Jung, S.S. Kindermann, A.J. Bell, and T.J. Sejnowski, "Analysis of fMRI data by blind separation into independent spatial components," Human Brain Mapping, vol. 6, no. 3, 1998, pp. $160-88$.

13. M.J. McKeown and T.J. Sejnowski, "Independent Component Analysis of fMRI Data: Examining the Assumptions," Human Brain Mapping, vol. 6, 1998, pp. 368-372.

14. M. Naganawa, Y. Kimura, K. Ishii, K. Oda, K. Ishiwata, and A. Matani, "Extraction of a Plasma Time-Activity Curve From Dynamic Brain PET Images Based on Independent Component Analysis," IEEE Transactions on Biomedical Engineering, 2005, pp. 1-10.

15. H.J. Park, J.J. Kim, T. Youn, D.S. Lee, M.C. Lee, and J.S. Kwon, "Independent component model for cognitive functions of multiple subjects using [15O]H2O PET images," Human Brain Mapping., vol. 18, no. 4, 2003, pp. 284-95.

16. P. Switzer and A.A. Green, Min/max autocorrelation factors for multivariate spatial imagery, Technical Report, Dept. of Statistics, Stanford University, 1984. 